\title{
A case of the "cellular variant" of focal segmental glomerulosclerosis with acute renal failure that remitted completely with oral steroid administration
}

\author{
Gaku Konno • Go Someya • Kazuma Sekine • \\ Takuya Nishino $\cdot$ Shinya Kawamoto
}

Received: 14 January 2012/Accepted: 8 May 2012/Published online: 19 June 2012

(C) Japanese Society of Nephrology 2012

\begin{abstract}
A 73-year-old man with systemic edema and oliguria, which appeared within several days, was referred to our hospital. Urinalysis showed massive proteinuria, and the blood examination results indicated nephrotic syndrome. Renal biopsy revealed the existence of focal segmental glomerulosclerosis (FSGS), and further investigation indicated that the microscopic features were consistent with the "cellular variant (CELL)" according to the Columbia Classification. After biopsy, $40 \mathrm{mg}$ per day of oral prednisolone was administered daily. With steroid therapy, the abnormal values of the blood examination and urinalysis gradually reached normal, and complete remission was achieved on day 43 after initiating steroid administration. Microscopic investigation by follow-up renal re-biopsy revealed several sclerosing glomeruli; however, other intact glomeruli had no endothelial proliferation, which is a hallmark of CELL. The patient was discharged after biopsy and observed as an outpatient, with maintenance of clinical remission.
\end{abstract}

Keywords Focal segmental glomerulosclerosis . Columbia Classification - Cellular variant - Acute renal failure $\cdot$ Nephrotic syndrome $\cdot$ Oral steroid administration

G. Konno · G. Someya · K. Sekine · T. Nishino · S. Kawamoto Department of Internal Medicine, Nippon Kokan Hospital, 1-2-1 Kokan-dori, Kawasaki-ku, Kawasaki,

Kanagawa 210-0852, Japan

S. Kawamoto $(\bowtie)$

Department of Nephrology, Dokkyo Medical University

Koshigaya Hospital, 2-1-50 Minami-koshigaya, Koshigaya,

Saitama 343-8555, Japan

e-mail: kwmt@dokkyomed.ac.jp

\section{Introduction}

Focal segmental glomerulosclerosis is characterized by partial segmental sclerosis of the glomeruli and is often associated with nephrotic syndrome [1, 2]. In 2004, the new Columbia Classification was proposed based on the histopathological characteristics of the affected glomeruli [3]. Subsequent research revealed that each variant classified by this criteria showed different treatment responsiveness and prognosis. Consequently, the Columbia Classification may be useful as a predictor of the clinical course of FSGS. In spite of its usefulness, the number of reported cases based on this categorization has not been sufficient in the 8 years since the Columbia Classification was published. Moreover, because CELL is the least described variant of FSGS, the entity of CELL is still unclear. By reporting this FSGS CELL variant case in which the patient responded to steroid therapy, we here emphasize the significance of the pathological diagnosis based on the Columbia Classification and the necessity to accumulate further clinical cases.

Case report

A 73-year-old man was admitted to our hospital with generalized edema and oliguria that had appeared within a week. He had never been diagnosed with abnormal renal function, and the serum creatinine $(\mathrm{sCr})$ level had been $1.01 \mathrm{mg} / \mathrm{dl}$ on blood examination 2 months prior to admission. His past history included a previous cerebral infarct with no sequelae and idiopathic sudden sensorineural hearing impairment. Neither hypertension nor diabetes mellitus had been found previously. His medications included $200 \mathrm{mg}$ cilostazol, $50 \mathrm{mg}$ clopidogrel sulfate, $75 \mathrm{mg}$ difenidol hydrochloride, and $30 \mathrm{mg}$ oxazolam. 
On physical examination, his height was $168.8 \mathrm{~cm}$ and body weight $82.0 \mathrm{~kg}$ (which had increased to $7 \mathrm{~kg}$ more than normal), and he had high blood pressure at $162 / 88 \mathrm{mmHg}$. He showed prominent pitting edema in his face and extremities. The hematological data revealed hemoglobin of $12.7 \mathrm{~g} / \mathrm{dl}$, leukocyte count of $5750 / \mu \mathrm{l}$, platelet count of $217000 / \mu \mathrm{l}$, and C-reactive protein of $1.33 \mathrm{mg} / \mathrm{dl}$. Blood chemistry results showed total protein of $4.9 \mathrm{~g} / \mathrm{dl}$, serum albumin (Alb) of $2.20 \mathrm{~g} / \mathrm{dl}$, total cholesterol of $262 \mathrm{mg} / \mathrm{dl}$, and LDL of $193 \mathrm{mg} / \mathrm{dl}$. The blood urea nitrogen and sCr levels were $83 \mathrm{mg} / \mathrm{dl}$ and $6.95 \mathrm{mg} / \mathrm{dl}$, respectively. An arterial blood specimen on room air showed $\mathrm{pH} 7.35, \mathrm{pO}_{2} 75 \mathrm{mmHg}, \mathrm{pCO}_{2} 32 \mathrm{mmHg}$, and $\mathrm{HCO}_{3} 17.7 \mathrm{mmol} / \mathrm{l}$.

Serum IgG, IgA, IgM, and complement levels (C3, C4, $\mathrm{CH} 50)$ were within normal range. He was negative for antistreptolysin O (ASO) antibody, anti-streptokinase (ASK) antibody, anti-glomerular basement membrane (GBM) antibody, PR3-ANCA, and MPO-ANCA. Antinuclear antibody was positive at a titer of 1:160 by fluorescent antibody examination, and both homogenous and speckled patterns were detected. Serological markers for hepatitis B and C virus, HIV 1/2, and Treponema pallidum were negative.

The 24-h urinary protein and sodium excretions were $8.9 \mathrm{~g} /$ day and $11 \mathrm{mEq} /$ day, respectively. Urinary occult blood was detected by dipstick, but few red blood cells were observed in the sediment per high-power field. The selectivity index was 0.32 , and the excretion fraction of sodium (FENa) was $0.61 \%$.

Chest radiography showed increased vascular shadow, but no pleural effusion or mass. Abdominal ultrasonography revealed that the kidneys were of normal size, sustained vascular flow in the cortex, and had no significant distension of the bilateral renal pelvis or ascites.

An ultrasound-guided needle renal biopsy was performed on hospital day 10. The specimen consisted of two cores of renal parenchyma with seven glomeruli; four glomeruli were intact, two of which were collapsed and globally sclerotic (Fig. 1). The remainder showed segmental infiltration of foam cells into capillaries, endothelial proliferation, and adhesion of the glomerulus to Bowman's capsule and shrinkage of podocytes. The renal interstitium showed necrotic tubular epithelium and $40 \%$ tubulointerstitial damage. Immunofluorescence analysis revealed no deposition of $\operatorname{IgG}, \operatorname{IgA}, \operatorname{IgM}$, or $\mathrm{C} 3 \mathrm{c}$ in the glomeruli. On electron microscopy, the foot processes of the podocytes effaced diffusely (not shown). These findings were consistent with the "cellular variant" of focal segmental glomerulosclerosis based on the Columbia Classification.

For the edema and oliguria, $40 \mathrm{mg}$ intravenous furosemide injection was started on hospital day 4; however, urinary excretion continued to be $500 \mathrm{ml}$ per day; $25 \mathrm{mg}$ of losartan potassium administration was started on the same day as an anti-hypertensive drug, but after a week, the losartan dose was increased to $50 \mathrm{mg}$, and $10 \mathrm{mg}$ of cilnidipine was added because of sustained hypertension; $10 \mathrm{mg}$ of atorvastatin was started on hospital day 5 for dyslipidemia.

After the renal biopsy, administration of $40 \mathrm{mg} /$ day prednisolone (PSL) was started. For a week after the treatment was initiated, the urinary protein excretion and $\mathrm{sCr}$ level decreased markedly, followed by elevation of the plasma Alb level. The daily urine volume rose gradually and reached $2000 \mathrm{ml}$ on day 13 after starting steroid administration. Also, there was striking clinical improvement.

Urinary protein excretion fell below $1 \mathrm{~g} /$ day, and the FENa value reached a maximum of $2.69 \%$ on day 22 . Subsequently, a daily dose of $30 \mathrm{mg} /$ day PSL was maintained. Diuretics and anti-hypertensive drugs were tapered off with symptomatic improvement. The FENa value reached trough levels at $0.34 \%$ on day 39 and then increased again. On day 43, complete remission was achieved (Fig. 2). The final $\mathrm{sCr}$ and LDL values were 1.29 and $102 \mathrm{mg} / \mathrm{dl}$, and the patient's body weight fell to $63 \mathrm{~kg}$ (20 kg lost before treatment).

Renal re-biopsy was performed on day 51 to confirm the effectiveness of steroid therapy. The specimen consisted of three cores of renal parenchyma with eight glomeruli, five of which were globally sclerotic, accompanied by neighboring tubular damage. About $25 \%$ of the interstitium showed renal tubule injury with fibrotic changes (Fig. 3). Another showed only minimal change and no evidence of FSGS. Evidence of proliferation and duplication of basement membranes was not seen.

Soon after biopsy, the patient was discharged and has been followed up as an outpatient with tapered PSL administration, and clinical remission has been sustained.

\section{Discussion}

Focal segmental glomerulosclerosis (FSGS) is a clinicopathological syndrome characterized by nephrotic-range proteinuria and segmental sclerosis of the glomerulus. The etiology of FSGS is still unclear, but a defect of the podocytes is strongly suspected [1, 2].

Up until now, FSGS has generally been considered a steroid-resistant nephropathy, but recent histopathological research has revealed that this is not an accurate understanding. D'Agati et al. [3] proposed the new, mutually exclusive Columbia Classification in 2004, and according to this, FSGS is subdivided into five variants: collapsing (COLL), glomerular tip lesions (GTL), cellular (CELL), perihilar (PH), and not otherwise specified (NOS). Further investigation revealed that COLL has the worst prognosis $(\mathrm{CR}+\mathrm{PR} 13.2 \%)$ and GTL the highest responsiveness to 


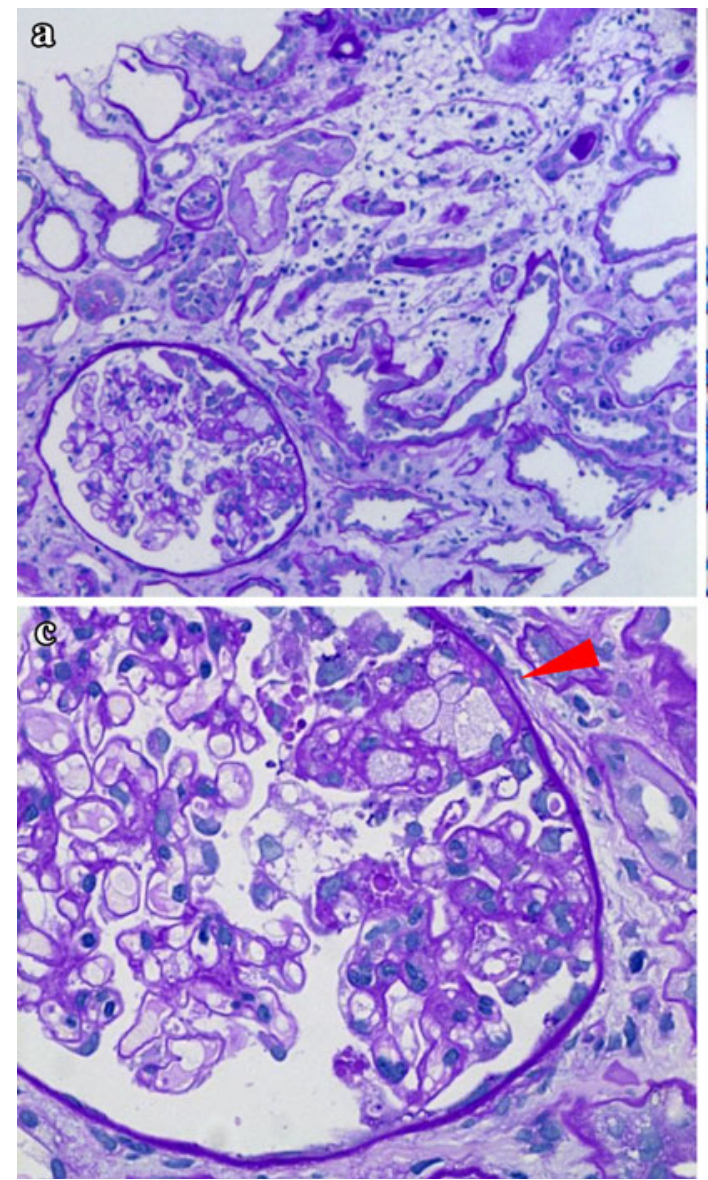

Fig. 1 Microscopic findings of the renal biopsy before steroid administration. a The glomerulus is partially sclerosed, and the interstitium is infiltrated by inflammatory cells, PAS stain $\times 4$. b Partially sclerosing glomerulus. Masson-trichrome stain $\times 10$.

steroid therapy (CR + PR $75.8 \%$ ) [4]. Therefore, determination of the subtype by renal biopsy in the early stage of FSGS is crucial for predicting the prognosis of each patient.

Microscopically, CELL is defined by the presence of at least one glomerulus with segmental expansion of the glomerular tuft involving more than $25 \%$ of the tuft by endocapillary hypercellularity, often with foam cells, with or without hyperplasia of the overlying visceral epithelial cells [4]. The responsiveness of CELL to treatment is intermediate (CR + PR 44.4\%), between COLL and GTL; however, the other clinical characteristics are still unclear.

Three large independent studies based on the Columbia Classification are known so far [4-6]. Some of these results are summarized in Table 1 . According to these, the majority of CELL cases present characteristics of nephrotic syndrome (UP $9.5 \pm 1.2 \mathrm{~g}, 8.9 \pm 2.3 \mathrm{~g}$, and $16 \pm 15 \mathrm{~g} / \mathrm{day}$; serum Alb $2.1 \pm 0.2 \mathrm{~g}, 2.84 \pm 0.32 \mathrm{~g}$, and $2.8 \pm 0.9 \mathrm{~g} / \mathrm{dl}$, respectively). However, the interval to biopsy $(4.3 \pm 0.9$ months,

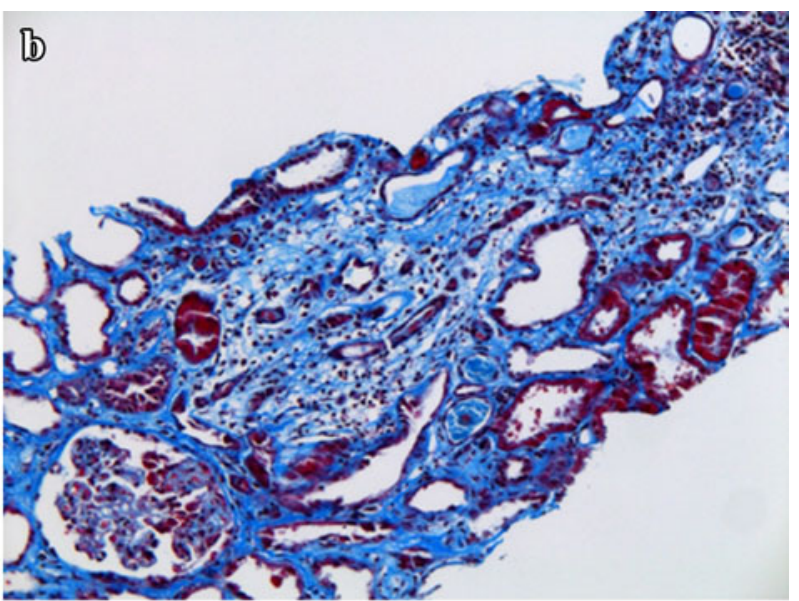

c High magnification of the glomerulus. Foam cells infiltrating into the capillaries (arrow) and endothelial proliferation are seen. The glomerulus is partially adherent to Bowman's capsule, PAS stain $\times 40$

$18.7 \pm 5.4$ months, and not shown, respectively) and serum Cr level $(1.9 \pm 0.3 \mathrm{mg}, 1.58 \pm 0.18 \mathrm{mg}$, and $2.5 \pm 1.7$ $\mathrm{mg} / \mathrm{dl}$, respectively) are not consistent with our case. As mentioned previously, the onset was sudden, and massive systemic edema and hypertension appeared within 7 days. The sCr level was extremely high $(6.95 \mathrm{mg} / \mathrm{dl})$ in comparison with these reports, and the duration of symptoms before biopsy was less than 1 month. In other words, our case can be called atypical "fulminant" FSGS.

What caused the acute deterioration of renal function in this patient? We speculate that the FENa value was one of the keys to the answer. Prior to steroid therapy, the FENa values were below $1 \%$, which is a strong indicator of prerenal factor involvement in the development of acute kidney injury in this case. Indeed, the primary injured region was the podocytes in FSGS, and this means acute intrarenal kidney injury in this context, but nephrotic-range proteinuria based on glomerular deterioration leads to a decrease of the colloid osmotic pressure. Then water escapes into the interstitium, and intravascular volume 
Fig. 2 Clinical course. Serum $\mathrm{Cr}$ level and urinary protein excretion decreased markedly after steroid administraion. Interestingly, the FENa shift was biphasic in response to prednisolone administration
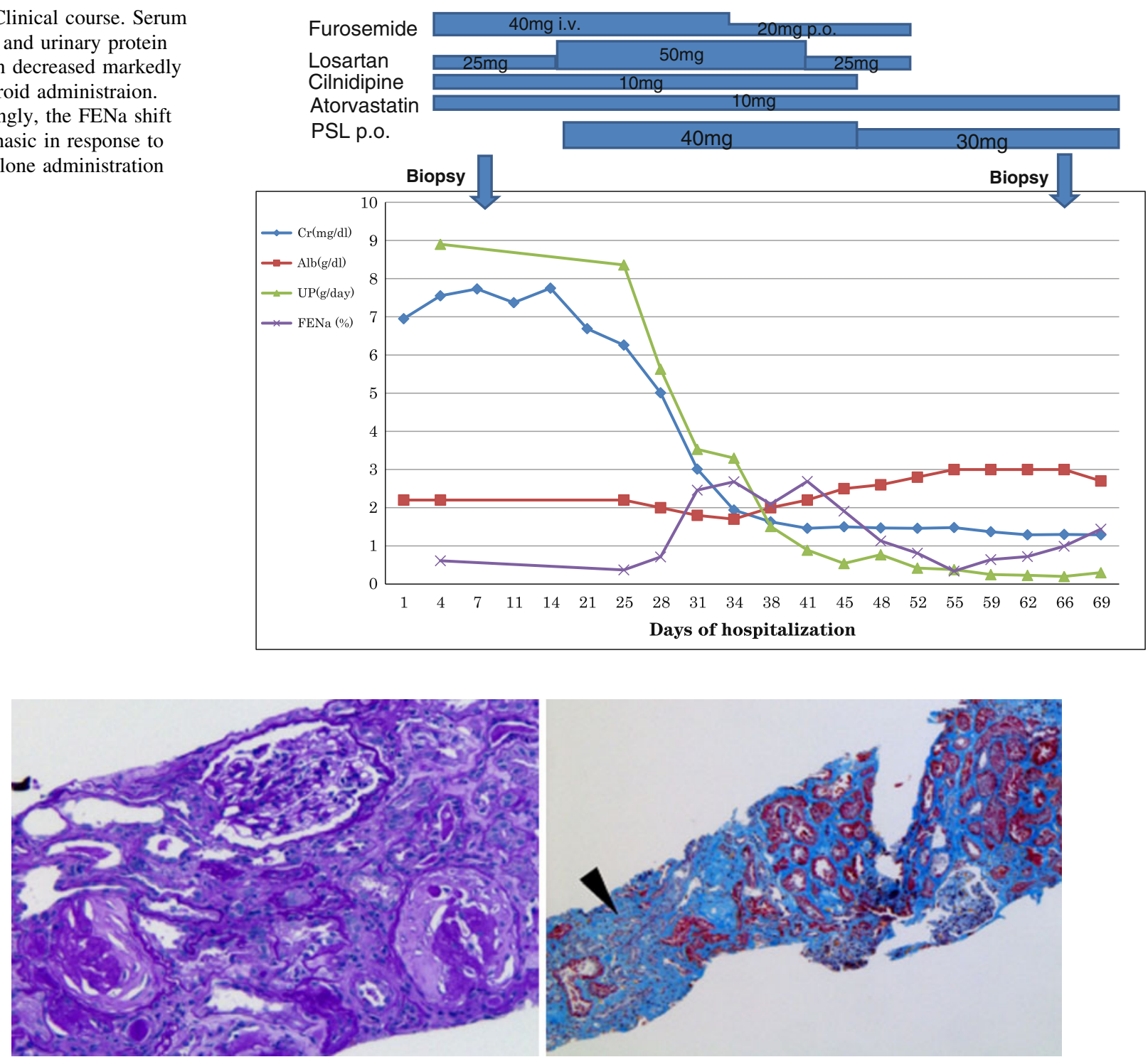

Fig. 3 Microscopic findings of renal biopsy after remission. Sclerosed glomeruli with tubular damage (arrow) can be observed; however, the intact glomerulus has no characteristics of FSGS

Table 1 Clinical features of the FSGS cellular variant reported in three previous studies

\begin{tabular}{lcccccc}
\hline Number & Age (years) & Serum Cr $(\mathrm{mg} / \mathrm{dl})$ & Serum Alb $(\mathrm{g} / \mathrm{dl})$ & UP $(\mathrm{g} / \mathrm{day})$ & Remission rate $(\%)$ & Reference \\
\hline 6 & $45 \pm 13$ & $2.5 \pm 1.7$ & $2.8 \pm 0.9$ & $16 \pm 15$ & 33 & 4 \\
22 & $48.8 \pm 4.9$ & $1.9 \pm 0.3$ & $2.1 \pm 0.2$ & $9.5 \pm 1.2$ & 44.4 & 5 \\
11 & $44.1 \pm 5.3$ & $1.58 \pm 0.18$ & $2.84 \pm 0.32$ & $8.9 \pm 2.3$ & 60 & 6 \\
\hline
\end{tabular}

depletion can develop. Consequently, it could be that the acuity of onset and development in our FSGS case was based on simultaneous, concomitant involvement of intrarenal and pre-renal factors.

Seven days after starting steroid administration, the FENa value rose abruptly, coinciding with increased urine excretion. This may indicate fluid re-filling into the intravascular space because of recovery of the epithelial function because of the immunosuppressive effect of the steroid. However, the high FENa value indicates insufficient sodium reabsorption. The FENa value began to fall on day 20 , which coincided with the recovery of the renal 
tubules. These FENa dynamics suggest that glomerular healing might precede the interstitial recovery with steroid therapy.

Although 7 years have passed since the Columbia Classification was published, subsequent case reports and analyses based on this classification are insufficient. According to our research, no case of fulminant FSGS with acute renal failure has been reported previously. Further investigation, not only pathologically but also clinically, is required to elucidate the entity of FSGS.

\section{References}

1. Dijkman H, Smeets B, van der Laak J, Steenbergen E, Wetzels J. The parietal epithelial cell is crucially involved in human idiopathic focal segmental glomerulosclerosis. Kidney Int. 2005;68:1562-72.
2. Gbadegesin R, Lavin P, Foreman J, Winn M. Pathogenesis and therapy of focal segmental glomerulosclerosis: an update. Pediatr Nephrol. 2011;26:1001-15.

3. D'Agati VD, Fogo AB, Bruijn JA, Jennette JC. Pathologic classification of focal segmental glomerulosclerosis: a working proposal. Am J Kidney Dis. 2004;43:368-82.

4. Thomas DB, Franceschini N, Hogan SL, Ten Holder S, Jennette CE, Falk RJ, et al. Clinical and pathologic characteristics of focal segmental glomerulosclerosis pathologic variants. Kidney Int. 2006;69:920-6.

5. Stokes MB, Valeri AM, Markowitz GS, D'Agati VD. Cellular focal segmental glomerulosclerosis: Clinical and pathologic features. Kidney Int. 2006;70:1783-92.

6. Taneda S, Honda K, Uchida K, Nitta K, Yumura W, Oda H, et al. Histological heterogeneity of glomerular segmental lesions in focal segmental glomerulosclerosis. Int Urol Nephrol. doi: 10.1007/s11255-011-9932-y. 